Pamela R. Hughes, MD; Carol H. Hungerford, DO; Kevin N. Jensen, DO

Family Medicine Residency Clinic (Dr. Hughes) and Family Health Clinic (Dr. Hungerford), Mike O'Callaghan Military Medical Center, Las Vegas, NV; Ear Nose and Throat Specialists of Alaska, Wasilla (Dr. Jensen)

-ypamela.r.hughes4.mil@ mail.mil

The authors reported no potential conflict of interest relevant to this article.

The opinions and assertions contained herein are those of the authors and are not to be construed as official or as reflecting the views of the US Air Force Medical Department, the US Air Force at large, or the US Department of Defense.

\title{
Acute rhinosinusitis: When to prescribe an antibiotic
}

\author{
Yes, the majority of antibiotics prescribed for acute \\ rhinosinusitis are unnecessary, but when should you \\ prescribe one and which one(s) should you use?
}

\section{PRACTICE RECOMMENDATIONS}

> Reserve antibiotics for patients who meet diagnostic criteria for acute bacterial rhinosinusitis (ABRS).

Patients must have purulent nasal drainage that is accompanied by either nasal obstruction or facial pain/ pressure/fullness and EITHER symptoms that persist without improvement for at least 10 days OR symptoms that worsen within 10 days of initial improvement ("double sickening"). A

> Offer watchful waiting and delay antibiotics for up to 7 days after diagnosing ABRS in a patient if adequate access to followup is available; otherwise, treat with amoxicillin (with or without clavulanate) for 5 to 10 days. A

Strength of recommendation (SOR)

A Good-quality patient-oriented evidence

B Inconsistent or limited-quality patient-oriented evidence

C Consensus, usual practice, opinion, disease-oriented evidence, case series
A n estimated 30 million cases of acute rhinosinusitis (ARS) occur every year in the United States. ${ }^{1}$ More than $80 \%$ of people with ARS are prescribed antibiotics in North America, accounting for $15 \%$ to $20 \%$ of all antibiotic prescriptions in the adult outpatient setting. ${ }^{2,3}$ Many of these prescriptions are unnecessary, as the most common cause of ARS is a virus. ${ }^{4,5}$ Evidence consistently shows that symptoms of ARS will resolve spontaneously in most patients and that only those patients with severe or prolonged symptoms require consideration of antibiotic therapy. ${ }^{1,2,4,6}$ Nearly half of all patients will improve within 1 week and two-thirds of patients will improve within 2 weeks without the use of antibiotics. ${ }^{7}$ In children, only about $6 \%$ to $7 \%$ presenting with upper respiratory symptoms meet the criteria for acute bacterial rhinosinusitis (ABRS), ${ }^{8}$ which we'll detail in a bit. For most patients, treatment should consist of symptom management. ${ }^{5}$

But what about the minority who require antibiotic therapy? This article reviews how to evaluate patients with ARS, identify those who require antibiotics, and prescribe the most appropriate antibiotic treatment regimens.

\section{Diagnosis: Distinguishing} viral from bacterial disease

ARS is defined as the sudden onset of purulent nasal discharge plus either nasal blockage or facial pressure/pain lasting $<4$ weeks. ${ }^{3,9}$ Additional signs and symptoms may include postnasal drip, a reduced sense of smell, sinus tenderness to palpation, and maxillary toothaches. ${ }^{10,11}$

ARS may be viral or bacterial in etiology, with the most common bacterial organisms being Streptococcus pneumoniae, Haemophilus influenzae, and Moraxella catarrhalis. ${ }^{1,3,5}$ The most common viral causes are influenza, parainfluenza, and rhinovirus. Approximately $90 \%$ to $98 \%$ of cases of ARS are viral $^{6,11}$; only about $0.5 \%$ to $2 \%$ of viral rhinosinusitis episodes are 
complicated by bacterial infection. ${ }^{1,10-12}$

Diagnose ABRS when symptoms of ARS fail to improve after 10 days or symptoms of ARS worsen within 10 days after initial improvement ("double sickening").1,11 Symptoms that are significantly associated with ABRS are unilateral sinus pain and reported maxillary pain. The presence of facial or dental pain correlates with ABRS but does not identify the specific sinus involved. ${ }^{1}$

There isn't good correlation between patients saying they have sinusitis and actually having it. ${ }^{13}$ A 2019 meta-analysis by Ebell et $\mathrm{al}^{14}$ reported that based on limited data, the overall clinical impression, fetid odor on the breath, and pain in the teeth are the best individual clinical predictors of ABRS.

As recommended by the Infectious Disease Society of America (IDSA), a diagnosis of ABRS is also reasonable in patients who present with severe symptoms at the onset. ${ }^{6}$ Although there is no consensus about what constitutes "severe symptoms," they are often described as a temperature $\geq 102^{\circ} \mathrm{F}\left(39^{\circ} \mathrm{C}\right)$ plus 3 to 4 days of purulent nasal drainage. ${ }^{1,4,6}$

Additional symptoms of ABRS may include cough, fatigue, decreased or lack of sense of smell (hyposmia or anosmia), and ear pressure. ${ }^{10}$ Another sign of "double sickening" is the development of a fever after several days of symptoms. ${ }^{1,9,15}$ Viral sinusitis typically lasts 5 to 7 days with a peak at days 2 to $3^{1,15}$ If symptoms continue for 10 days, there is a $60 \%$ chance of bacterial sinusitis, although some viral rhinosinusitis symptoms persist for $>14$ days. ${ }^{1,5}$ Beyond 4 to 12 weeks, sinusitis is classified as subacute or chronic. ${ }^{3}$

\section{Physical exam findings and the limited roles of imaging and labs}

Common physical exam findings associated with the diagnosis of ABRS include altered speech indicating nasal obstruction; edema or erythema of the skin indicating congested capillaries; tenderness to palpation over the cheeks or upper teeth; odorous breath; and purulent drainage from the nose or in the posterior pharynx.

In a study by Hansen et $\mathrm{al}^{13}(\mathrm{~N}=174)$, the only sign that showed significant association with ABRS (diagnosed by sinus aspiration or lavage) was unilateral tenderness of the maxillary sinuses. The presence of purulent drainage in the nose or posterior pharynx also has significant diagnostic value, as it predicts the presence of bacteria on antral aspiration. ${ }^{1}$ Purulent discharge in the pharynx is associated with a higher likelihood of benefit from antibiotic therapy compared to placebo (number needed to treat $[\mathrm{NNT}]=8$ ). ${ }^{16}$ However, colored nasal discharge indicates the presence of neutrophils-not bacteria-and does not predict the likelihood of bacterial sinus infection. ${ }^{14,17}$ Therefore, the history and physical exam should focus on location of pain (sinus and/or teeth), duration of symptoms, presence of fever, change in symptom severity, attempted home therapies, sinus tenderness on exam, breath odor, and purulent drainage seen in the nasal cavity or posterior pharynx. ${ }^{13,14}$

Radiographic imaging has no role in the diagnosis or treatment of uncomplicated ABRS because viral and bacterial etiologies have similar radiographic appearances. Additionally, employing radiologic imaging would increase health care costs by at least 4-fold. ${ }^{5,6,8,17}$ The American Academy of Otolaryngology-Head and Neck Surgery (AAO-HNS) clinical practice guidelines recommend against radiographic imaging for patients who meet the diagnostic criteria for ABRS unless concern exists for a complication or an alternate diagnosis is suspected. ${ }^{1}$ Computed tomography (CT) imaging of the sinuses may be warranted in patients with severe headaches, facial swelling, cranial nerve palsies, or bulging of the eye (proptosis), all of which indicate a potential complication of ABRS. ${ }^{1}$

- Laboratory evaluations. ABRS is a clinical diagnosis; therefore, routine lab work, such as a white blood cell count, C-reactive protein (CRP) level, and/or erythrocyte sedimentation rate (ESR), are not indicated unless an alternate diagnosis is suspected. ${ }^{1,5,13,18,19}$

In one study, CRP > $10 \mathrm{mg} / \mathrm{L}$ and ESR $>10 \mathrm{~mm} / \mathrm{h}$ were the strongest individual predictors of purulent antral puncture aspirate or positive bacterial culture of aspirate, which is considered diagnostic for ABRS. ${ }^{20}$ However, CRP and ESR by themselves are not adequate
Approximately $90 \%$ to $98 \%$ of cases of acute rhinosinusitis are viral; only about $0.5 \%$ to $2 \%$ of viral rhinosinusitis episodes are complicated by bacterial infection. 
TABLE 1

\section{Diagnostic criteria for acute bacterial rhinosinusitis}

\begin{tabular}{l|l}
\hline AAO-HNS & $\begin{array}{l}\text { ARS symptoms (purulent nasal discharge plus either nasal obstruction or facial pain/pressure) plus } \\
1 \text { of the following: } \\
\text { Persistent symptoms for } 10 \text { days after onset without improvement } \\
\text { OR } \\
\text { Acute worsening of symptoms after an initial improvement ("double sickening") }\end{array}$ \\
\hline IDSA $^{6}$ & $\begin{array}{l}\text { Presence of ARS symptoms with any of the following: } \\
\text { - Severe features (temperature } \geq 102^{\circ} \mathrm{F}\left[39^{\circ} \mathrm{C}\right] \text { and facial pain) for at least } 3 \text { days } \\
\text { - "Double sickening" }\end{array}$ \\
\hline
\end{tabular}

AAO-HNS, American Academy of Otolaryngology-Head and Neck Surgery; ARS, acute rhinosinusitis; IDSA, Infectious Diseases Society of America.

to diagnose ABRS. ${ }^{20}$ This study developed a clinical decision rule that used symptoms, signs, and laboratory values to rate the likelihood of ABRS as being either low, moderate, or high. However, this clinical decision rule has not been prospectively validated.

Thus, CRP and ESR elevations can support the diagnosis of ABRS, but the low sensitivity of these tests precludes their use as a screening tool for ABRS. ${ }^{14,18}$ Studies by Ebell ${ }^{19}$ and Huang ${ }^{21}$ have shown some benefit to dipstick assay of nasal secretions for the diagnosis of ABRS, but this method is not validated or widely used. ${ }^{19,21}$

\section{Treatment: From managing} symptoms to prescribing antibiotics Overprescribing antibiotics for ARS is a prominent health care issue. In fact, 5 of 9 placebo-controlled studies showed that most people improve within 2 weeks regardless of antibiotic use $(\mathrm{N}=1058){ }^{3}$ Therefore, weigh the decision to treat ABRS with antibiotics against the risk for potential adverse reactions and within the context of antibiotic stewardship. ${ }^{2,9,12,22-24}$ Consider antibiotics only if patients meet the diagnostic criteria for ABRS (TABLE $1^{1,6}$ ) or, occasionally, for patients with severe symptoms upon presentation, such as a temperature $\geq 102^{\circ} \mathrm{F}\left(39^{\circ} \mathrm{C}\right)$ plus purulent nasal discharge for 3 to 4 days. ${ }^{1}$ The most commonly reported adverse effects of antibiotics are gastrointestinal in nature and include nausea, vomiting, and diarrhea., ${ }^{2,9}$

I Symptomatic management for both
ARS and ABRS is recommended as first-line therapy; it should be offered to patients before making a diagnosis of ABRS..$^{1,5,9,25}$ Consider using analgesics, topical intranasal steroids, and/or nasal saline irrigation to alleviate symptoms and improve quality of life. ${ }^{1,5,25} \mathrm{In}$ terventions with questionable or unproven efficacy include the use of antihistamines, systemic steroids, decongestants, and mucolytics, but they may be considered on an individual basis. ${ }^{1}$ A systematic review found that topical nasal steroids relieved facial pain and nasal congestion in patients with rhinitis and acute sinusitis $(\mathrm{NNT}=14) .{ }^{1,26}$

Even after diagnosing ABRS, clinicians should offer watchful waiting and symptomatic therapies as long as patients have adequate access to follow-up (TABLE 2,1,15 FIGURE $^{1,6}$ ). Antibiotic therapy can then be initiated if symptoms do not improve after an additional 7 days of watchful waiting or if symptoms worsen at any time. It is reasonable to give patients a prescription to keep on hand to be used if symptoms worsen, with instructions to notify the provider if antibiotics are started. ${ }^{1}$

Intibiotic therapy. The rationale for treating ABRS with antibiotics is to expedite recovery and prevent complications such as periorbital or orbital cellulitis, meningitis, frontal osteomyelitis, cavernous sinus thrombosis, and other serious illness. ${ }^{27}$ Antibiotic treatment is associated with a shorter duration of symptoms (NNT $=19)$ but an increased risk of adverse events $(\mathrm{NNH}=8))^{7,19}$

Amoxicillin with or without clavulanate 
TABLE 2

\section{Recommended treatment for acute bacterial rhinosinusitis}

\begin{tabular}{|c|c|}
\hline $\begin{array}{l}\text { Watchful waiting with symptomatic } \\
\text { treatment }^{1}\end{array}$ & $\begin{array}{l}\text { - Analgesics (acetaminophen or NSAIDs) } \\
\text { - Topical intranasal steroids } \\
\text { - Nasal saline irrigation }\end{array}$ \\
\hline First-line antibiotics for adults ${ }^{1}$ & - Amoxicillin with or without clavulanate for 5-10 d \\
\hline Penicillin-allergic adult patients ${ }^{1}$ & $\begin{array}{l}\text { - Doxycycline } \\
\text { moxpiratory fluoroquinolone (levofloxacin or } \\
\text { - Non-type I hypersensitivity reaction to penicillin: } \\
\text { clindamycin plus third-generation oral cephalosporin } \\
\text { (cefixime or cefpodoxime) }\end{array}$ \\
\hline Children $^{15}$ & $\begin{array}{l}\text { - Amoxicillin with or without clavulanate for 5-10 d } \\
\text { Penicillin allergic: } \\
\text { - Non-type } 1 \text { reaction: cefpodoxime, cefuroxime, or } \\
\text { cefdinir } \\
\text { - Clindamycin (or linezolid) plus cefixime } \\
\text { - Levofloxacin }\end{array}$ \\
\hline Adult treatment failure $^{1}$ & $\begin{array}{l}\text { - Amoxicillin/clavulanate if initially given amoxicillin alone } \\
\text { OR } 1 \text { of the following: } \\
\text { - Doxycycline } \\
\text { - Respiratory fluoroquinolone (levofloxacin or } \\
\text { moxifloxacin) } \\
\text { - Clindamycin plus third-generation cephalosporin }\end{array}$ \\
\hline
\end{tabular}

NSAIDs, nonsteroidal anti-inflammatory drugs.

for 5 to 10 days is first-line antibiotic therapy for most adults with ABRS. ${ }^{1,3,5,8,9,11}$ Per AAO-HNS, the "justification for amoxicillin as first-line treatment relates to its safety, efficacy, low cost, and narrow microbiologic spectrum." ${ }^{1}$ Amoxicillin may be dosed $500 \mathrm{mg}$ tid for 5 to 10 days. Amoxicillin/clavulanate (Augmentin) is recommended for patients with comorbid conditions or with increased risk of bacterial resistance. Dosing for amoxicillin/clavulanate is $500 / 125 \mathrm{mg}$ tid or $875 / 125 \mathrm{mg}$ bid for 5 to 10 days. Duration of therapy should be determined by the severity of symptoms. ${ }^{5}$

I For penicillin-allergic patients, doxycycline or a respiratory fluoroquinolone (levofloxacin or moxifloxacin) is considered first-line treatment. ${ }^{1,6}$ Doxycycline is preferred because of its narrower spectrum and fewer adverse effects than the fluoroquinolones. Fluoroquinolones should be reserved for patients who fail first-line treatment and are penicillin allergic. ${ }^{1}$ Because of the high rates of resistance among $S$ pneumoniae and H influenzae, macrolides, trimethoprim/sulfamethoxazole (TMP/SMX), and cephalosporins are not recommended as first-line therapy. ${ }^{1,5}$

I How antibiotic options compare. A Cochrane review of 54 studies comparing different antibiotics showed no antibiotic was superior. ${ }^{3}$ Of the 54 studies, 6 studies $(\mathrm{N}=1887)$ were pooled to compare cephalosporins to amoxicillin/clavulanate at 7 to 15 days. The findings indicated a statistically significant difference for amoxicillin/clavulanate with a relative risk (RR) of 1.37 (confidence interval [CI], 1.04-1.8). ${ }^{3}$ However, none of these 6 studies were graded as having a low risk of bias; therefore, confidence in this finding was deemed limited due to the quality of included studies. The failure rate for cephalosporins was $12 \%$ vs $8 \%$ for amoxicillin/clavulanate. ${ }^{3}$

Treatment failure is considered when a patient has not improved by Day 7 after ABRS di-

\section{Diagnose acute bacterial rhinosinusitis when symptoms of acute rhinosinusitis fail to improve after 10 days or symptoms of ARS worsen within 10 days after initial improvement.}




\section{FIGURE}

\section{Treatment of acute bacterial rhinosinusitis}

$>$

Colored nasal discharge indicates the presence of neutrophils-not bacteria-and does not predict the likelihood of bacterial sinus infection.

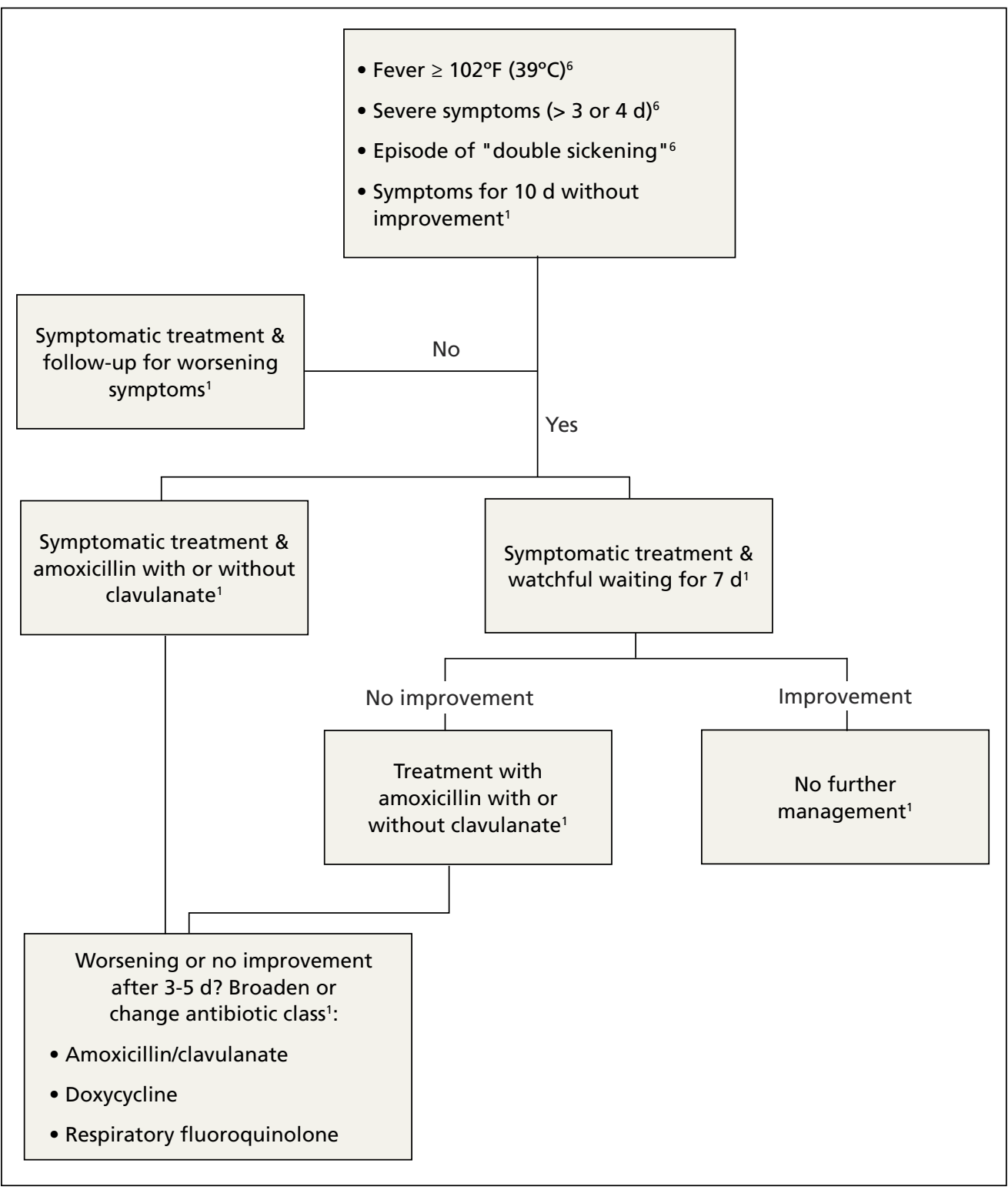

agnosis (with or without medication) or when symptoms worsen at any time. If watchful waiting was chosen and a safety net prescription was provided, the antibiotics should be filled and started. If no antibiotic was prescribed at the time watchful waiting commenced, the patient should return for further evaluation and be started on antibiotics. If antibiotics were prescribed initially for severe symptoms, a change in antibiotic therapy is indicated, and a broader-spectrum antibiotic should be cho- sen. If amoxicillin was prescribed, the patient should be switched to amoxicillin/clavulanate, doxycycline, a respiratory fluoroquinolone, or a combination of clindamycin plus a third-generation cephalosporin. ${ }^{1}$

\section{Diagnosis and management of pediatric patients}

Diagnosis of ABRS in children is defined as an acute upper respiratory infection (URI) 
accompanied by persistent nasal discharge, daytime cough for $\geq 10$ days without improvement, an episode of "double sickening," or severe onset with a temperature $\geq 102^{\circ} \mathrm{F}$ and purulent nasal discharge for 3 days. ${ }^{15}$

Initial presentations of viral URIs and ABRS are almost identical; thus, persistence of symptoms is key to diagnosis. ${ }^{6}$ Nasal discharge tends to appear several days after initial symptoms manifest for viral infections including influenza. In children $<5$ years of age, the most common complication involves the orbit. ${ }^{15}$ Orbital complications generally manifest with eye pain and/or periorbital swelling and may be accompanied by proptosis or decreased functioning of extraocular musculature. The differential diagnosis for orbital complications includes cavernous sinus thrombosis, orbital cellulitis/abscess, subperiosteal abscess, and inflammatory edema. $^{27,28}$ Intracranial complications are also possible with severe ABRS. ${ }^{12}$

Radiology studies are not recommended for the initial diagnosis of ABRS in children, as again, imaging does not differentiate between viral and bacterial etiologies. However, in children with complications such as orbital or cerebral involvement, a contrast-enhanced CT scan of the paranasal sinuses is indicated. ${ }^{15}$

I Antibiotic therapy is indicated in children with a diagnosis of severe ABRS or in cases of "double sickening." Clinicians may consider watchful waiting for 3 additional days before initiating antibiotics in patients meeting criteria for ABRS. Amoxicillin with or without clavulanate is the antibiotic of choice. $^{15}$

For penicillin-allergic children without a history of anaphylactoid reaction, treatment with cefpodoxime, cefdinir, or cefuroxime is appropriate. For children with a history of anaphylaxis, treatment with a combination of clindamycin (or linezolid) and cefixime is indicated. Alternatively, a fluoroquinolone such as levofloxacin may be used, but adverse effects and emerging resistance limit its use..$^{15}$

\section{Specialist referral}

Referral to Otolaryngology is indicated for patients with $>3$ episodes of clinically diag- nosed bacterial sinusitis in 1 year, evidence of fungal disease (which is outside the scope of this article), immunocompromised status, or a persistent temperature $\geq 102^{\circ} \mathrm{F}$ despite antibiotic therapy. Also consider otolaryngology referral for patients with a history of sinus surgery. ${ }^{2,5,6}$

JFP

\section{CORRESPONDENCE}

Pamela R. Hughes, Family Medicine Residency Clinic, Mike O'Callaghan Military Medical Center, 4700 Las Vegas Boulevard North, Nellis AFB, NV 89191; pamela.r.hughes4. mil@mail.mil.

\section{REFERENCES}

1. Rosenfeld RM, Piccirillo JF, Chandrasekhar SS, et al. Clinical practice guideline (update): adult sinusitis. Otolaryngol Head Neck Surg. 2015;152(2 suppl):S1-S39.

2. Fokkens WJ, Hoffmans R, Thomas M. Avoid prescribing antibiotics in acute rhinosinusitis. BMJ. 2014;349:g5703.

3. Ahovuo-Saloranta A, Rautakorpi UM, Borisenko OV, et al. Antibiotics for acute maxillary sinusitis in adults. Cochrane Database Syst Rev. 2014:CD000243.

4. Burgstaller, JM, Steurer J, Holzmann D, et al. Antibiotic efficacy in patients with a moderate probability of acute rhinosinusitis: a systematic review. Eur Arch Otorhinolaryngol. 2016;273:1067 1077.

5. Aring AM, Chan MM. Current concepts in adult acute rhinosinusitis. Am Fam Physician. 2016;94:97-105.

6. Chow AW, Benninger MS, Brook I, et al. IDSA clinical practice guideline for acute bacterial rhinosinusitis in children and adults. Clin Infect Dis. 2012;54:e72-e112.

7. Lemiengre MB, van Driel ML, Merenstein D, et al. Antibiotics for acute rhinosinusitis in adults. Cochrane Database Syst Rev. 2018:CD006089.

8. Harris AM, Hicks LA, Qaseem A. Appropriate antibiotic use for acute respiratory tract infection in adults: advice for high-value care from the American College of Physicians and the Centers for Disease Control and Prevention. Ann Intern Med. 2016;164:425434.

9. Sng WJ, Wang DY. Efficacy and side effects of antibiotics in the treatment of acute rhinosinusitis: a systematic review. Rhinology. 2015;53:3-9.

10. Benninger M, Segreti J. Is it bacterial or viral? Criteria for distinguishing bacterial and viral infections. J Fam Pract. 2008;57(2 suppl):S5-S11.

11. Sharma P, Finley R, Weese S, et al. Antibiotic prescriptions for outpatient acute rhinosinusitis in Canada, 2007-2013. PLoS One. 2017;12:e0181957.

12. Pynnonen MA, Lynn S, Kern HE, et al. Diagnosis and treatment of acute sinusitis in the primary care setting: a retrospective cohort. Laryngoscope. 2015;125:2266-2272.

13. Hansen JG, Schmidt H, Rosborg J, et al. Predicting acute maxillary sinusitis in a general practice population. BMJ 1995;311:233-236.

14. Ebell MH, McKay B, Dale, A, et al. Accuracy of signs and symptoms for the diagnosis of acute rhinosinusitis and acute bacterial rhinosinusitis. Ann Fam Med. 2019;17:164-172.

15. Wald ER, Applegate KE, Bordley C, et al. Clinical practice guideline for the diagnosis and management of acute bacterial sinusitis in children aged 1 to 18 years. Pediatrics. 2013;132:e262-e280.

16. Young J, De Sutter A, Merenstein D, et al. Antibiotics for adults with clinically diagnosed acute rhinosinusitis: a meta-analysis of individual patient data. Lancet. 2008;371:908-914.

17. Smith SS, Ference EH, Evan CT, et al. The prevalence of bacteria infection in acute rhinosinusitis: a systematic review and metaanalysis. Laryngoscope. 2015;125:57-69

18. Autio TJ, Koskenkorva T, Koivunen P, et al. Inflammatory biomarkers during bacterial acute rhinosinusitis. Curr Allergy Asthma Rep. 2018;18:13.

19. Ebell MH, McKay B, Guilbault R, et al. Diagnosis of acute rhino-
Weigh the decision to treat acute bacterial rhinosinusitis with antibiotics against the risk for potential adverse reactions and within the context of antibiotic stewardship. 
sinusitis in primary care: a systematic review of test accuracy. $\mathrm{Br}$ Gen Pract. 2016;66:e612-e632.

20. Ebell MH, Hansen JG. Proposed clinical decision rules to diagnose acute rhinosinusitis among adults in primary care. Ann Fam Med. 2017;15:347-354.

21. Huang SW, Small PA. Rapid diagnosis of bacterial sinusitis in patients using a simple test of nasal secretions. Allergy Asthma Proc. 2008;29:640-643.

22. Smith SS, Evans CT, Tan BK, et al. National burden of antibiotic use for adult rhinosinusitis. J Allergy Clin Immunol. 2013; 132

23. Barlam TF, Soria-Saucedo R, Cabral HJ, et al. Unnecessary antibiotics for acute respiratory tract infections: association with care setting and patient demographics. Open Forum Infect Dis.
2016;3:1-7.

24. Fleming-Dutra KE, Hersh AL, Shapiro DJ, et al. Prevalence of inappropriate antibiotic prescriptions among US ambulatory care visits, 2010-2011. JAMA. 2016;315:1864-1873.

25. Garbutt JM, Banister C, Spitznagel E, et al. Amoxicillin for acute rhinosinusitis: a randomized controlled trial. JAMA 2012;307:685-692.

26. Zalmanovici Trestioreanu A, Yaphe J. Intranasal steroids for acute sinusitis. Cochrane Database Syst Rev. 2013:CD005149.

27. Abzug MJ. Acute sinusitis in children: do antibiotics have any role? J Infect. 2014;68 (suppl 1):S33-S37.

28. Williams JW Jr, Simel DL, Roberts L, et al. Clinical evaluation for sinusitis. Making the diagnosis by history and physical examination. Ann Intern Med. 1992;117:705-710.

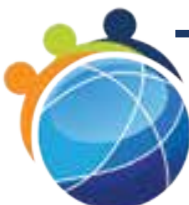

\section{MEDJOBNETWORK, COM}

Physician o NP/PA Career Center

\section{The first mobile job board for Physicians, NPs, and PAs}

\section{Mobile Job Searches - access MedJobNetwork.com} on the go from your smartphone or tablet

Advanced Search Capabilities - search for jobs by specialty, job title, geographic location, employer, and more

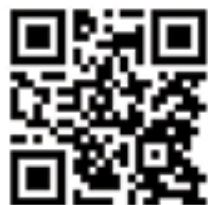

Scan this $Q R$ code

to access the mobile version

of MedJobNetwork.com 